NISSUNA UMANA INVESTIGAZIONE SI PUO DIMANDARE VERA SCIENZIA S'ESSA NON PASSA PER LE MATEMATICHE DIMOSTRAZIONI LEONARDO DA VINCI

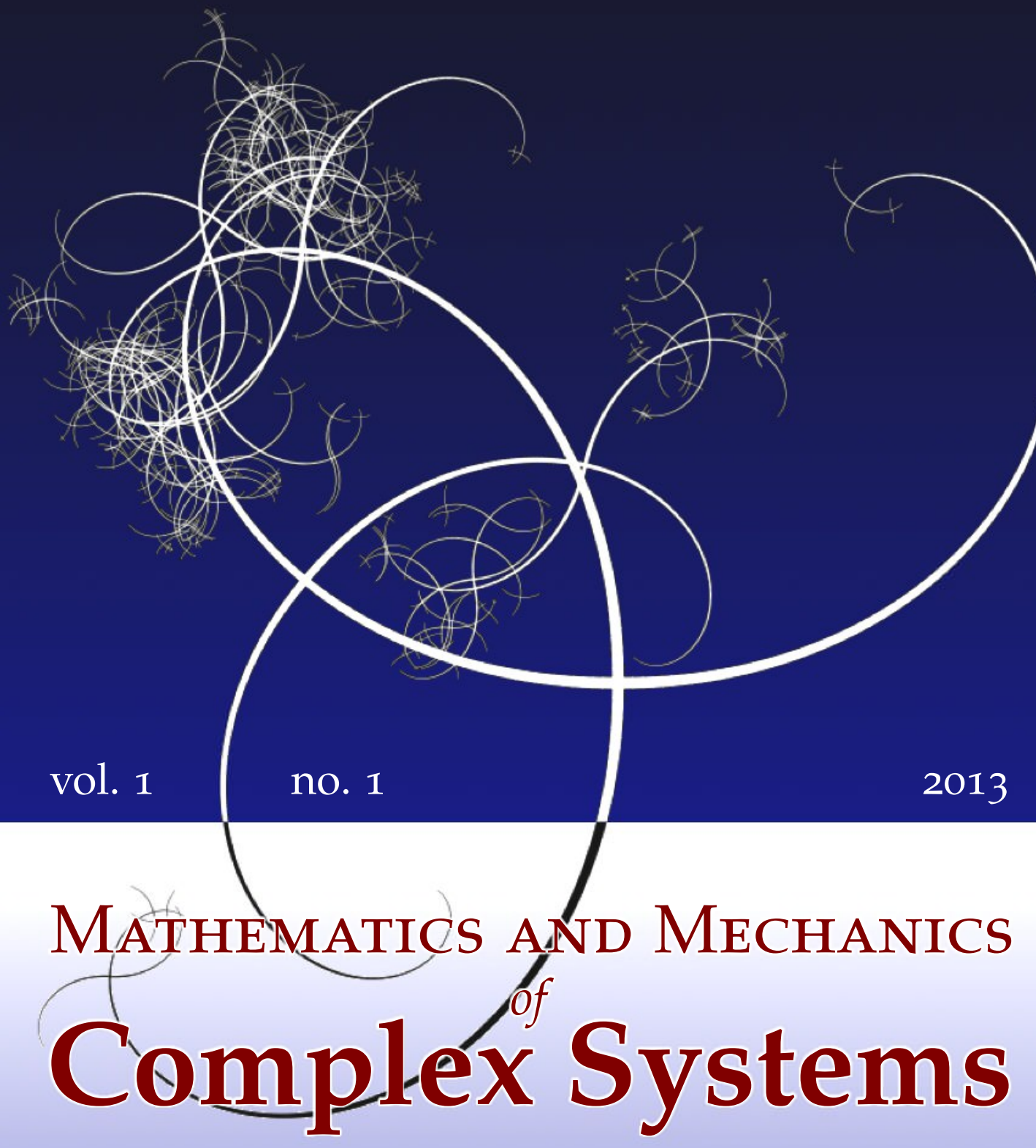

Xu Wang And Peter Schiavone

DISLOCATIONS, IMPERFECT INTERFACES

AND INTERFACE CRACKS IN ANISOTROPIC ELASTICITY FOR QUASICRYSTALS 


\title{
DISLOCATIONS, IMPERFECT INTERFACES AND INTERFACE CRACKS IN ANISOTROPIC ELASTICITY FOR QUASICRYSTALS
}

\author{
Xu Wang And Peter Schiavone
}

\begin{abstract}
We derive the detailed structures of the $6 \times 6$ matrices $N_{i}$ and $N_{i}^{(-1)}(i=1,2,3)$ in the Stroh formalism of anisotropic elasticity for quasicrystals. All six matrices are expressed explicitly in terms of the sixty-six reduced elastic compliances. The Green's functions for bi-quasicrystals are also obtained. Next, we derive compliant and stiff interface models in anisotropic quasicrystalline bimaterials. It is observed that the phonon normal traction is always continuous across the stiff interface. Finally we present the asymptotic fields associated with a tractionfree, semi-infinite interface crack in anisotropic quasicrystalline bimaterials and solve the collinear interface crack problem. The interface crack-tip field consists of three two-dimensional oscillatory singularities which are evaluated via the introduction of three complex stress intensity factors.
\end{abstract}

\section{Introduction}

The theory of anisotropic elasticity for crystals has been actively investigated for more than half a century; see, for example, [Lekhnitskii 1950; Eshelby et al. 1953; Stroh 1958; Willis 1964; Willis 1970; Willis 1971; Clements 1971; Barnett and Lothe 1973; Suo 1990; Suo et al. 1992; Gao et al. 1992; Ting 1986; Ting 1988; Ting 1996; Ru 2001; Cheng and Reddy 2002; Ting and Schiavone 2010; Wang and Pan 2010]. As pointed out in [Ting 1996], the Stroh formalism [1958], which is based on Stroh eigenvalues and eigenvectors, allows for an elegant and powerful analysis of two-dimensional deformations of anisotropic crystalline solids where as many as fifteen elastic constants are involved. The beauty of the Stroh formalism has indeed been observed by various researchers; see, for example, [Suo 1990; Ru 2001; Cheng and Reddy 2002; Lazar and Kirchner 2005; Wang and Pan 2010].

Quasicrystalline structures were first reported in [Shechtman et al. 1984]. The generalized anisotropic elasticity for quasicrystals developed in [Hu et al. 2000] requires that anisotropic quasicrystals have as many as one hundred and twenty elastic constants. Even for the study of two-dimensional deformations of quasicrystals,

\section{Communicated by David Steigmann.}

MSC2010: primary 74B05, 74E10; secondary 74E15.

Keywords: quasicrystal, anisotropic elasticity, Stroh formalism, dislocation, interface crack. 
sixty-six pertinent elastic constants remain. Ding et al. [1995] extended the original six-dimensional Stroh formalism for crystals by developing a twelve-dimensional version in order to study dislocation problems in quasicrystals.

Outline of paper. In Section 2 we present the Stroh formalism for two-dimensional deformations in anisotropic quasicrystals. In Section 3, we derive the detailed structures of $N_{i}$ and $N_{i}^{(-1)}(i=1,2,3)$ appearing in the Stroh formalism for quasicrystals through the introduction of sixty-six reduced elastic compliances $S_{i j}=S_{j i}$ $(i, j=1 \ldots 15$ and $i, j \neq 3,9,10,14)$. In Section 4 , the Green's functions for a line force and dislocation located

(1) in a homogeneous quasicrystal,

(2) along the interface of bi-quasicrystals, and

(3) in one of two bonded quasicrystalline half-planes

are investigated. Based on the structures obtained for $N_{i}(i=1,2,3)$, we then develop in Section 5 two imperfect interface models in anisotropic quasicrystalline bimaterials. Finally, in Section 6, we derive the near-tip field of an interface crack in anisotropic bi-quasicrystals consisting of three two-dimensional coupled oscillatory singularities. Three complex intensity factors $K_{1}, K_{2}$ and $K_{3}$ are introduced to quantify the near-tip field. Also studied in Section 6 is the collinear interface crack problems in anisotropic bi-quasicrystals following the decoupling method proposed in [Suo 1990] and [Suo et al. 1992].

\section{The Stroh formalism}

In a fixed rectangular coordinate system $x_{i}(i=1,2,3)$, let $u_{i}$ and $w_{i}$ be the phonon and phason displacements, $\sigma_{i j}\left(\sigma_{i j}=\sigma_{j i}\right)$ and $H_{i j}\left(H_{i j} \neq H_{j i}\right)$ be the phonon and phason stresses in an anisotropic quasicrystalline material. The stress-strain law and the equations of equilibrium are [Hu et al. 2000]:

$$
\begin{aligned}
\sigma_{i j} & =C_{i j k l} u_{k, l}+R_{i j k l} w_{k, l}, & \sigma_{i j, j} & =0, \\
H_{i j} & =R_{k l i j} u_{k, l}+K_{i j k l} w_{k, l}, & H_{i j, j} & =0,
\end{aligned}
$$

where the comma denotes differentiation, $C_{i j k l}$ are the elastic constants in the phonon field, $K_{i j k l}$, are the elastic constants in the phason field and $R_{i j k l}$ are the phonon-phason coupling constants. In addition $C_{i j k l}, R_{i j k l}$ and $K_{i j k l}$ possess the following symmetry:

$$
C_{i j k l}=C_{j i k l}=C_{k l i j}=C_{i j l k}, \quad R_{i j k l}=R_{j i k l}, \quad K_{i j k l}=K_{k l i j} .
$$


For two-dimensional deformations in which $u_{i}$ and $w_{i}$ depend only on $x_{1}$ and $x_{2}$, the general solutions can be expressed as

$$
\begin{aligned}
\boldsymbol{u} & =\left[\begin{array}{llllll}
u_{1} & u_{2} & u_{3} & w_{1} & w_{2} & w_{3}
\end{array}\right]^{T}=\boldsymbol{A} \boldsymbol{f}(z)+\overline{\boldsymbol{A}} \overline{\boldsymbol{f}(z)}, \\
\boldsymbol{\Phi} & =\left[\begin{array}{llllll}
\Phi_{1} & \Phi_{2} & \Phi_{3} & \Psi_{1} & \Psi_{2} & \Psi_{3}
\end{array}\right]^{T}=\boldsymbol{B} \boldsymbol{f}(z)+\overline{\boldsymbol{B}} \overline{\boldsymbol{f}(z)},
\end{aligned}
$$

where

$$
\begin{aligned}
& \boldsymbol{A}=\left[\begin{array}{llllll}
\boldsymbol{a}_{1} & \boldsymbol{a}_{2} & \boldsymbol{a}_{3} & \boldsymbol{a}_{4} & \boldsymbol{a}_{5} & \boldsymbol{a}_{6}
\end{array}\right], \quad \boldsymbol{B}=\left[\begin{array}{llllll}
\boldsymbol{b}_{1} & \boldsymbol{b}_{2} & \boldsymbol{b}_{3} & \boldsymbol{b}_{4} & \boldsymbol{b}_{5} & \boldsymbol{b}_{6}
\end{array}\right], \\
& \boldsymbol{f}(z)=\left[\begin{array}{llllll}
f_{1}\left(z_{1}\right) & f_{2}\left(z_{2}\right) & f_{3}\left(z_{3}\right) & f_{4}\left(z_{4}\right) & f_{5}\left(z_{5}\right) & f_{6}\left(z_{6}\right)
\end{array}\right]^{T}, \\
& z_{i}=x_{1}+p_{i} x_{2}, \quad \operatorname{Im}\left\{p_{i}\right\}>0 \quad(i=1, \ldots, 6),
\end{aligned}
$$

with

$$
\begin{gathered}
{\left[\begin{array}{ll}
\boldsymbol{N}_{1} & \boldsymbol{N}_{2} \\
\boldsymbol{N}_{3} & \boldsymbol{N}_{1}^{T}
\end{array}\right]\left[\begin{array}{l}
\boldsymbol{a}_{i} \\
\boldsymbol{b}_{i}
\end{array}\right]=p_{i}\left[\begin{array}{c}
\boldsymbol{a}_{i} \\
\boldsymbol{b}_{i}
\end{array}\right] \quad(i=1, \ldots, 6),} \\
\boldsymbol{N}_{1}=-\boldsymbol{T}^{-1} \boldsymbol{R}^{T}, \quad \boldsymbol{N}_{2}=\boldsymbol{T}^{-1}, \quad \boldsymbol{N}_{3}=\boldsymbol{R} \boldsymbol{T}^{-1} \boldsymbol{R}^{T}-\boldsymbol{Q},
\end{gathered}
$$

$$
\boldsymbol{Q}=\left[\begin{array}{llllll}
C_{11} & C_{16} & C_{15} & R_{11} & R_{19} & R_{15} \\
C_{16} & C_{66} & C_{56} & R_{61} & R_{69} & R_{65} \\
C_{15} & C_{56} & C_{55} & R_{51} & R_{59} & R_{55} \\
R_{11} & R_{61} & R_{51} & K_{11} & K_{19} & K_{15} \\
R_{19} & R_{69} & R_{59} & K_{19} & K_{99} & K_{59} \\
R_{15} & R_{65} & R_{55} & K_{15} & K_{59} & K_{55}
\end{array}\right], \quad \boldsymbol{R}=\left[\begin{array}{llllll}
C_{16} & C_{12} & C_{14} & R_{16} & R_{12} & R_{17} \\
C_{66} & C_{26} & C_{46} & R_{66} & R_{62} & R_{67} \\
C_{56} & C_{25} & C_{45} & R_{56} & R_{52} & R_{57} \\
R_{61} & R_{21} & R_{41} & K_{16} & K_{12} & K_{17} \\
R_{69} & R_{29} & R_{49} & K_{69} & K_{29} & K_{79} \\
R_{65} & R_{25} & R_{45} & K_{56} & K_{25} & K_{57}
\end{array}\right],
$$

$$
\boldsymbol{T}=\left[\begin{array}{llllll}
C_{66} & C_{26} & C_{46} & R_{66} & R_{62} & R_{67} \\
C_{26} & C_{22} & C_{24} & R_{26} & R_{22} & R_{27} \\
C_{46} & C_{24} & C_{44} & R_{46} & R_{42} & R_{47} \\
R_{66} & R_{26} & R_{46} & K_{66} & K_{26} & K_{67} \\
R_{62} & R_{22} & R_{42} & K_{26} & K_{22} & K_{27} \\
R_{67} & R_{27} & R_{47} & K_{67} & K_{27} & K_{77}
\end{array}\right]
$$

The matrices $\boldsymbol{Q}$ and $\boldsymbol{T}$ are symmetric and positive definite.

In (7), we have adopted the contracted notation

$11 \leftrightarrow 1,22 \leftrightarrow 2,33 \leftrightarrow 3,23 \leftrightarrow 4,31 \leftrightarrow 5,12 \leftrightarrow 6,32 \leftrightarrow 7,13 \leftrightarrow 8,21 \leftrightarrow 9$.

In addition the stress function vector $\boldsymbol{\Phi}$ is defined, in terms of the phonon and phason stresses, by

$$
\sigma_{i 1}=-\Phi_{i, 2}, \quad \sigma_{i 2}=\Phi_{i, 1}, \quad H_{i 1}=-\Psi_{i, 2}, \quad H_{i 2}=\Psi_{i, 1} \quad(i=1,2,3) .
$$

The $6 \times 6$ matrices $\boldsymbol{A}$ and $\boldsymbol{B}$ satisfy the normalized orthogonal relationship 


$$
\left[\begin{array}{ll}
\boldsymbol{B}^{T} & \boldsymbol{A}^{T} \\
\overline{\boldsymbol{B}}^{T} & \overline{\boldsymbol{A}}^{T}
\end{array}\right]\left[\begin{array}{cc}
\boldsymbol{A} & \overline{\boldsymbol{A}} \\
\boldsymbol{B} & \overline{\boldsymbol{B}}
\end{array}\right]=\boldsymbol{I} .
$$

Therefore three real Barnett-Lothe tensors $\boldsymbol{S}, \boldsymbol{H}$ and $\boldsymbol{L}$ can be introduced:

$$
\boldsymbol{S}=\mathrm{i}\left(2 \boldsymbol{A} \boldsymbol{B}^{T}-\boldsymbol{I}\right), \quad \boldsymbol{H}=2 \mathrm{i} \boldsymbol{A} \boldsymbol{A}^{T}, \quad \boldsymbol{L}=-2 \mathrm{i} \boldsymbol{B} \boldsymbol{B}^{T} .
$$

Here $\boldsymbol{H}$ and $\boldsymbol{L}$ are positive definite matrices. It can also be easily checked that

$$
\left[\begin{array}{ll}
N_{1}^{(-1)} & N_{2}^{(-1)} \\
N_{3}^{(-1)} & N_{1}^{(-1)^{T}}
\end{array}\right]\left[\begin{array}{l}
a_{i} \\
b_{i}
\end{array}\right]=\frac{1}{p_{i}}\left[\begin{array}{l}
a_{i} \\
b_{i}
\end{array}\right] \quad(i=1, \ldots, 6),
$$

where

$$
\boldsymbol{N}_{1}^{(-1)}=-\boldsymbol{Q}^{-1} \boldsymbol{R}, \quad \boldsymbol{N}_{2}^{(-1)}=-\boldsymbol{Q}^{-1}, \quad \boldsymbol{N}_{3}^{(-1)}=\boldsymbol{T}-\boldsymbol{R}^{T} \boldsymbol{Q}^{-1} \boldsymbol{R} .
$$

In addition, both the $6 \times 6$ matrix $\boldsymbol{M}$ and its inverse $\boldsymbol{M}^{-1}$, defined by

$$
\boldsymbol{M}=-\mathrm{i} \boldsymbol{B} \boldsymbol{A}^{-1}=\boldsymbol{H}^{-1}+\mathrm{i} \boldsymbol{H}^{-1} \boldsymbol{S}, \quad \boldsymbol{M}^{-1}=\mathrm{i} \boldsymbol{A} \boldsymbol{B}^{-1}=\boldsymbol{L}^{-1}-\mathrm{i} \boldsymbol{S} \boldsymbol{L}^{-1},
$$

are positive definite Hermitian matrices. In this work we make use of a positive definite Hermitian matrix $\boldsymbol{N}$ involving bimaterial elastic constants and defined as

$$
\boldsymbol{N}=\boldsymbol{M}_{1}^{-1}+\overline{\boldsymbol{M}}_{2}^{-1}=\boldsymbol{L}_{1}^{-1}+\boldsymbol{L}_{2}^{-1}+\mathrm{i}\left(\boldsymbol{S}_{2} \boldsymbol{L}_{2}^{-1}-\boldsymbol{S}_{1} \boldsymbol{L}_{1}^{-1}\right) .
$$

It is clear from (7) that for two-dimensional deformations of quasicrystalline materials, there are in total sixty-six elastic constants: fifteen in the phonon field, twenty-one in the phason field and thirty phonon-phason coupling constants. In the next section we present the detailed structures of $\boldsymbol{N}_{i}$ and $N_{i}^{(-1)}(i=1,2,3)$.

\section{The structures of $N_{i}$ and $N_{i}^{(-1)}$}

Consider first the structure of $\boldsymbol{N}_{i}(i=1,2,3)$. Since the second column of $\boldsymbol{Q}$ is identical to the first column of $\boldsymbol{R}$, and the second row of $\boldsymbol{R}$ is identical to the first row of $\boldsymbol{T}$, we have

$$
N_{1}=\left[\begin{array}{cccccc}
* & -1 & * & * & * & * \\
* & 0 & * & * & * & * \\
* & 0 & * & * & * & * \\
* & 0 & * & * & * & * \\
* & 0 & * & * & * & * \\
* & 0 & * & * & * & *
\end{array}\right], \quad \boldsymbol{N}_{3}=\left[\begin{array}{cccccc}
* & 0 & * & * & * & * \\
0 & 0 & 0 & 0 & 0 & 0 \\
* & 0 & * & * & * & * \\
* & 0 & * & * & * & * \\
* & 0 & * & * & * & * \\
* & 0 & * & * & * & *
\end{array}\right],
$$

where $*$ denotes a possibly nonzero element. 
Next, we introduce the reduced elastic compliances $S_{i j}=S_{j i}(i, j=1 \ldots 15$ and $i, j \neq 3,9,10,14)$ such that

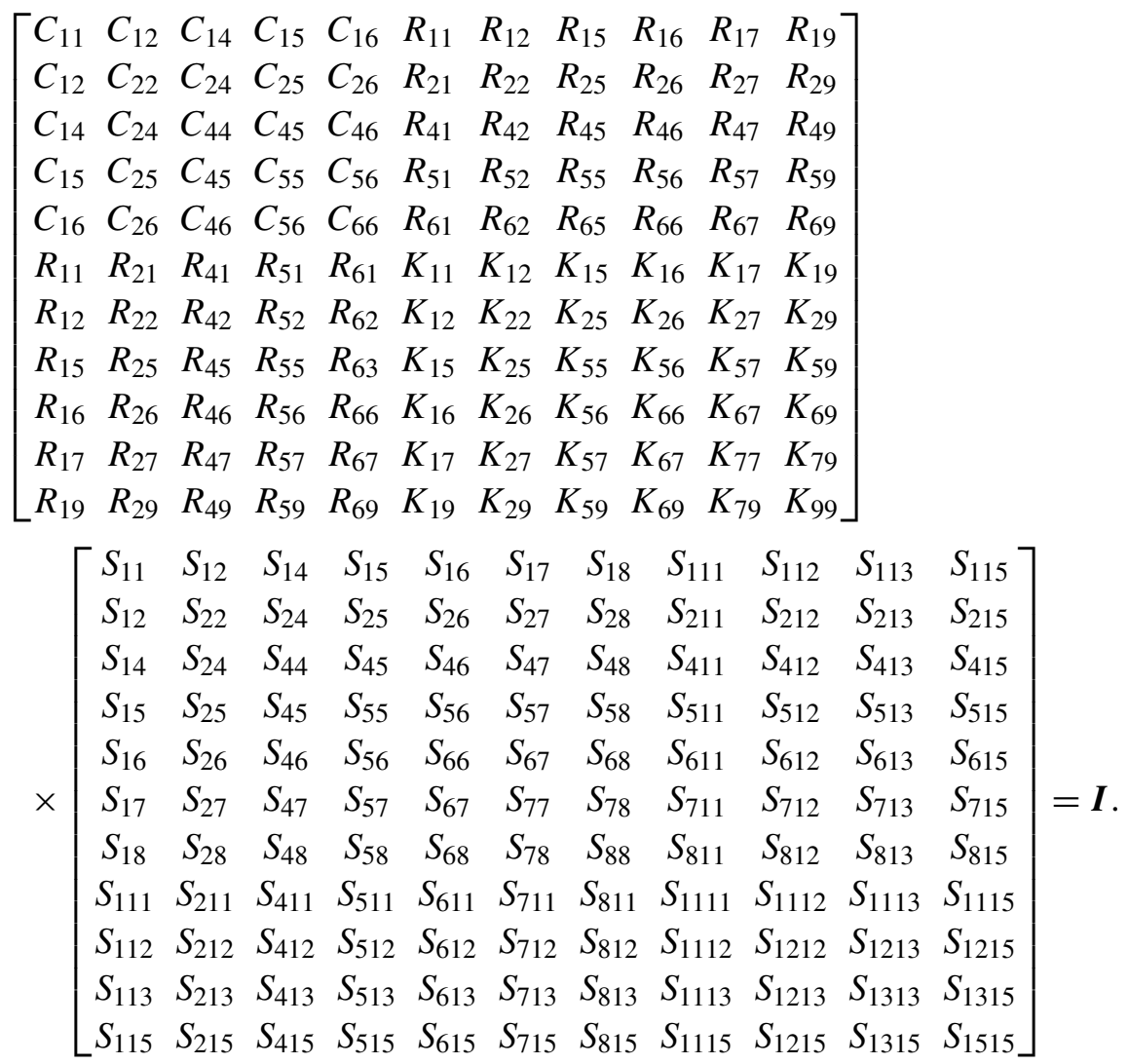

Remark. We adopt the convention that if three digits appear as subscripts of $S_{i j}$, the first digit is $i$ and the remaining two form $j$. If four digits appear in the subscripts of $S_{i j}$, the first two digits are $i$ and the remaining two will form $j$.

It can be easily deduced from (16) that

$$
\left[\begin{array}{ll}
\boldsymbol{Q} & \boldsymbol{R} \\
\boldsymbol{R}^{T} & \boldsymbol{T}
\end{array}\right]\left[\begin{array}{cc}
\boldsymbol{q}_{2} & \boldsymbol{r}_{2} \\
\boldsymbol{r}_{2}^{T} & \boldsymbol{t}
\end{array}\right]=\left[\begin{array}{cc}
\boldsymbol{I}-\boldsymbol{I}_{2} & \boldsymbol{I}_{12}^{T} \\
\mathbf{0} & \boldsymbol{I}
\end{array}\right]
$$

where

$$
\boldsymbol{q}_{2}=\left[\begin{array}{cccccc}
S_{11} & 0 & S_{15} & S_{17} & S_{115} & S_{111} \\
0 & 0 & 0 & 0 & 0 & 0 \\
S_{15} & 0 & S_{55} & S_{57} & S_{515} & S_{511} \\
S_{17} & 0 & S_{57} & S_{77} & S_{715} & S_{711} \\
S_{115} & 0 & S_{515} & S_{715} & S_{1515} & S_{1115} \\
S_{111} & 0 & S_{511} & S_{711} & S_{1115} & S_{1111}
\end{array}\right]
$$




$$
\begin{aligned}
& \boldsymbol{r}_{2}=\left[\begin{array}{cccccc}
S_{16} & S_{12} & S_{14} & S_{112} & S_{18} & S_{113} \\
0 & 0 & 0 & 0 & 0 & 0 \\
S_{56} & S_{25} & S_{45} & S_{512} & S_{58} & S_{513} \\
S_{67} & S_{27} & S_{47} & S_{712} & S_{78} & S_{713} \\
S_{615} & S_{215} & S_{415} & S_{715} & S_{815} & S_{1315} \\
S_{611} & S_{211} & S_{411} & S_{711} & S_{811} & S_{1113}
\end{array}\right] \\
& \boldsymbol{t}=\left[\begin{array}{cccccc}
S_{66} & S_{26} & S_{46} & S_{612} & S_{68} & S_{613} \\
S_{26} & S_{22} & S_{24} & S_{212} & S_{28} & S_{213} \\
S_{46} & S_{24} & S_{44} & S_{412} & S_{48} & S_{413} \\
S_{612} & S_{212} & S_{412} & S_{1212} & S_{812} & S_{1213} \\
S_{68} & S_{28} & S_{48} & S_{812} & S_{88} & S_{813} \\
S_{613} & S_{213} & S_{413} & S_{1213} & S_{813} & S_{1313}
\end{array}\right], \\
& \boldsymbol{I}_{12}=\left[\begin{array}{llllll}
0 & 1 & 0 & 0 & 0 & 0 \\
0 & 0 & 0 & 0 & 0 & 0 \\
0 & 0 & 0 & 0 & 0 & 0 \\
0 & 0 & 0 & 0 & 0 & 0 \\
0 & 0 & 0 & 0 & 0 & 0 \\
0 & 0 & 0 & 0 & 0 & 0
\end{array}\right], \quad \boldsymbol{I}_{2}=\operatorname{diag}\left[\begin{array}{llllll}
0 & 1 & 0 & 0 & 0 & 0
\end{array}\right] .
\end{aligned}
$$

After some algebraic manipulations, we finally arrive at

$$
\begin{aligned}
N_{3}=-\boldsymbol{q}_{2}^{-1}=\frac{-1}{\Delta}\left[\begin{array}{cccccc}
\widehat{W}_{11} & 0 & \widehat{W}_{12} & \widehat{W}_{13} & \widehat{W}_{14} & \widehat{W}_{15} \\
0 & 0 & 0 & 0 & 0 & 0 \\
\widehat{W}_{12} & 0 & \widehat{W}_{22} & \widehat{W}_{23} & \widehat{W}_{24} & \widehat{W}_{25} \\
\widehat{W}_{13} & 0 & \widehat{W}_{23} & \widehat{W}_{33} & \widehat{W}_{34} & \widehat{W}_{35} \\
\widehat{W}_{14} & 0 & \widehat{W}_{24} & \widehat{W}_{34} & \widehat{W}_{44} & \widehat{W}_{45} \\
\widehat{W}_{15} & 0 & \widehat{W}_{25} & \widehat{W}_{35} & \widehat{W}_{45} & \widehat{W}_{55}
\end{array}\right], \\
\boldsymbol{N}_{1}=\boldsymbol{r}_{2}^{T} \boldsymbol{q}_{2}^{-1}-\boldsymbol{I}_{12}=\left[\begin{array}{cccccc}
r_{6} & -1 & s_{6} & t_{6} & u_{6} & v_{6} \\
r_{2} & 0 & s_{2} & t_{2} & u_{2} & v_{2} \\
r_{4} & 0 & s_{4} & t_{4} & u_{4} & v_{4} \\
r_{12} & 0 & s_{12} & t_{12} & u_{12} & v_{12} \\
r_{8} & 0 & s_{8} & t_{8} & u_{8} & v_{8} \\
r_{13} & 0 & s_{13} & t_{13} & u_{13} & v_{13}
\end{array}\right], \\
\boldsymbol{N}_{2}=\boldsymbol{t}-\boldsymbol{r}_{2}^{T} \boldsymbol{q}_{2}^{-1} \boldsymbol{r}_{2}=\left[\begin{array}{cccccc}
\kappa_{66} & \kappa_{26} & \kappa_{46} & \kappa_{612} & \kappa_{68} & \kappa_{613} \\
\kappa_{26} & \kappa_{22} & \kappa_{24} & \kappa_{212} & \kappa_{28} & \kappa_{213} \\
\kappa_{46} & \kappa_{24} & \kappa_{44} & \kappa_{412} & \kappa_{48} & \kappa_{413} \\
\kappa_{612} & \kappa_{212} & \kappa_{412} & \kappa_{1212} & \kappa_{812} & \kappa_{1213} \\
\kappa_{68} & \kappa_{28} & \kappa_{48} & \kappa_{812} & \kappa_{88} & \kappa_{813} \\
\kappa_{613} & \kappa_{213} & \kappa_{413} & \kappa_{1213} & \kappa_{813} & \kappa_{1313}
\end{array}\right],
\end{aligned}
$$

where $\boldsymbol{q}_{2}^{-1}$ is the pseudo-inverse of $\boldsymbol{q}_{2}, \Delta$ is the determinant of 


$$
\boldsymbol{W}=\left[\begin{array}{ccccc}
S_{11} & S_{15} & S_{17} & S_{115} & S_{111} \\
S_{15} & S_{55} & S_{57} & S_{515} & S_{511} \\
S_{17} & S_{57} & S_{77} & S_{715} & S_{711} \\
S_{115} & S_{515} & S_{715} & S_{1515} & S_{1115} \\
S_{111} & S_{511} & S_{711} & S_{1115} & S_{1111}
\end{array}\right],
$$

$\widehat{\boldsymbol{W}}=\left[\widehat{W}_{i j}\right]$ is the cofactor matrix of $\boldsymbol{W}$, and

$$
\begin{aligned}
& r_{\alpha}=\frac{1}{\Delta}\left|\begin{array}{lllll}
S_{1 \alpha} & S_{5 \alpha} & S_{7 \alpha} & S_{15 \alpha} & S_{11 \alpha} \\
S_{15} & S_{55} & S_{57} & S_{515} & S_{511} \\
S_{17} & S_{57} & S_{77} & S_{715} & S_{711} \\
S_{115} & S_{515} & S_{715} & S_{1515} & S_{1115} \\
S_{111} & S_{511} & S_{711} & S_{115} & S_{1111}
\end{array}\right|, \quad s_{\alpha}=\frac{1}{\Delta}\left|\begin{array}{lllll}
S_{11} & S_{15} & S_{17} & S_{115} & S_{111} \\
S_{1 \alpha} & S_{5 \alpha} & S_{7 \alpha} & S_{15 \alpha} & S_{11 \alpha} \\
S_{17} & S_{57} & S_{77} & S_{715} & S_{711} \\
S_{115} & S_{515} & S_{715} & S_{1515} & S_{1115} \\
S_{111} & S_{511} & S_{711} & S_{1115} & S_{1111}
\end{array}\right|, \\
& t_{\alpha}=\frac{1}{\Delta}\left|\begin{array}{lllll}
S_{11} & S_{15} & S_{17} & S_{115} & S_{111} \\
S_{15} & S_{55} & S_{57} & S_{515} & S_{511} \\
S_{1 \alpha} & S_{5 \alpha} & S_{7 \alpha} & S_{15 \alpha} & S_{11 \alpha} \\
S_{115} & S_{515} & S_{715} & S_{1515} & S_{1115} \\
S_{111} & S_{511} & S_{711} & S_{1115} & S_{1111}
\end{array}\right|, \quad u_{\alpha}=\frac{1}{\Delta}\left|\begin{array}{lllll}
S_{11} & S_{15} & S_{17} & S_{115} & S_{111} \\
S_{15} & S_{55} & S_{57} & S_{515} & S_{511} \\
S_{17} & S_{57} & S_{77} & S_{715} & S_{711} \\
S_{1 \alpha} & S_{5 \alpha} & S_{7 \alpha} & S_{15 \alpha} & S_{11 \alpha} \\
S_{111} & S_{511} & S_{711} & S_{1115} & S_{1111}
\end{array}\right|, \\
& v_{\alpha}=\frac{1}{\Delta}\left|\begin{array}{lllll}
S_{11} & S_{15} & S_{17} & S_{115} & S_{111} \\
S_{15} & S_{55} & S_{57} & S_{515} & S_{511} \\
S_{17} & S_{57} & S_{77} & S_{715} & S_{711} \\
S_{115} & S_{515} & S_{715} & S_{1515} & S_{1115} \\
S_{1 \alpha} & S_{5 \alpha} & S_{7 \alpha} & S_{15 \alpha} & S_{11 \alpha}
\end{array}\right| \quad \quad(\alpha=6,2,4,12,8,13), \\
& \kappa_{\alpha \beta}=\frac{1}{\Delta}\left|\begin{array}{llllll}
S_{11} & S_{1 \alpha} & S_{15} & S_{17} & S_{115} & S_{111} \\
S_{1 \beta} & S_{\alpha \beta} & S_{5 \beta} & S_{7 \beta} & S_{15 \beta} & S_{11 \beta} \\
S_{15} & S_{5 \alpha} & S_{55} & S_{57} & S_{515} & S_{511} \\
S_{17} & S_{7 \alpha} & S_{57} & S_{77} & S_{715} & S_{711} \\
S_{115} & S_{15 \alpha} & S_{515} & S_{715} & S_{1515} & S_{1115} \\
S_{111} & S_{11 \alpha} & S_{511} & S_{711} & S_{1115} & S_{1111}
\end{array}\right| \quad \quad(\alpha, \beta=6,2,4,12,8,13) .
\end{aligned}
$$

In view of (6), $\boldsymbol{N}_{2}$ is positive definite, while (18) and the fact that $\boldsymbol{W}$ defined by (21) is positive definite result in $-\boldsymbol{N}_{3}$ being positive semidefinite.

We next derive the structure of $N_{i}^{(-1)}(i=1,2,3)$. It is not hard to check that

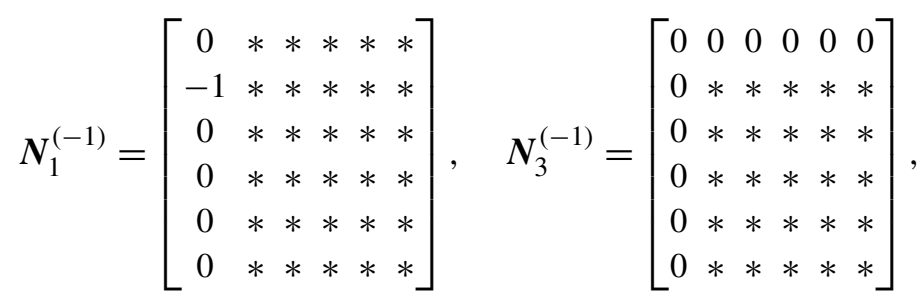


In addition, we have the identity

$$
\left[\begin{array}{ll}
\boldsymbol{Q} & \boldsymbol{R} \\
\boldsymbol{R}^{T} & \boldsymbol{T}
\end{array}\right]\left[\begin{array}{cc}
\boldsymbol{q} & \boldsymbol{r}_{1} \\
\boldsymbol{r}_{1}^{T} & \boldsymbol{t}_{1}
\end{array}\right]=\left[\begin{array}{cc}
\boldsymbol{I} & \mathbf{0} \\
\boldsymbol{I}_{12} & \boldsymbol{I}-\boldsymbol{I}_{1}
\end{array}\right],
$$

where

$$
\boldsymbol{q}=\left[\begin{array}{llllll}
S_{11} & S_{16} & S_{15} & S_{17} & S_{115} & S_{111} \\
S_{16} & S_{66} & S_{56} & S_{67} & S_{615} & S_{611} \\
S_{15} & S_{56} & S_{55} & S_{57} & S_{515} & S_{511} \\
S_{17} & S_{67} & S_{57} & S_{77} & S_{715} & S_{711} \\
S_{115} & S_{615} & S_{515} & S_{715} & S_{1515} & S_{1115} \\
S_{111} & S_{611} & S_{511} & S_{711} & S_{1115} & S_{1111}
\end{array}\right], \quad \boldsymbol{r}_{1}=\left[\begin{array}{llllll}
0 & S_{12} & S_{14} & S_{112} & S_{18} & S_{113} \\
0 & S_{26} & S_{46} & S_{612} & S_{68} & S_{613} \\
0 & S_{25} & S_{45} & S_{512} & S_{58} & S_{513} \\
0 & S_{27} & S_{47} & S_{712} & S_{78} & S_{713} \\
0 & S_{215} & S_{415} & S_{715} & S_{815} & S_{1315} \\
0 & S_{211} & S_{411} & S_{711} & S_{811} & S_{1113}
\end{array}\right],
$$

$$
\boldsymbol{t}_{1}=\left[\begin{array}{cccccc}
0 & 0 & 0 & 0 & 0 & 0 \\
0 & S_{22} & S_{24} & S_{212} & S_{28} & S_{213} \\
0 & S_{24} & S_{44} & S_{412} & S_{48} & S_{413} \\
0 & S_{212} & S_{412} & S_{1212} & S_{812} & S_{1213} \\
0 & S_{28} & S_{48} & S_{812} & S_{88} & S_{813} \\
0 & S_{213} & S_{413} & S_{1213} & S_{813} & S_{1313}
\end{array}\right], \quad \quad \boldsymbol{I}_{1}=\operatorname{diag}\left[\begin{array}{llllll}
1 & 0 & 0 & 0 & 0 & 0
\end{array}\right] .
$$

We can now arrive at

$$
\begin{aligned}
\boldsymbol{N}_{3}^{(-1)}=\boldsymbol{t}_{1}^{-1}=\frac{1}{\Delta^{\prime}} & {\left[\begin{array}{cccccc}
0 & 0 & 0 & 0 & 0 & 0 \\
0 & \widehat{W}_{11}^{\prime} & \widehat{W}_{12}^{\prime} & \widehat{W}_{13}^{\prime} & \widehat{W}_{14}^{\prime} & \widehat{W}_{15}^{\prime} \\
0 & \widehat{W}_{12}^{\prime} & \widehat{W}_{22}^{\prime} & \widehat{W}_{23}^{\prime} & \widehat{W}_{24}^{\prime} & \widehat{W}_{25}^{\prime} \\
0 & \widehat{W}_{13}^{\prime} & \widehat{W}_{23}^{\prime} & \widehat{W}_{33}^{\prime} & \widehat{W}_{34}^{\prime} & \widehat{W}_{35}^{\prime} \\
0 & \widehat{W}_{14}^{\prime} & \widehat{W}_{24}^{\prime} & \widehat{W}_{34}^{\prime} & \widehat{W}_{44}^{\prime} & \widehat{W}_{45}^{\prime} \\
0 & \widehat{W}_{15}^{\prime} & \widehat{W}_{25}^{\prime} & \widehat{W}_{35}^{\prime} & \widehat{W}_{45}^{\prime} & \widehat{W}_{55}^{\prime}
\end{array}\right], } \\
\boldsymbol{N}_{1}^{(-1)}=\boldsymbol{r}_{1} \boldsymbol{t}_{1}^{-1}-\boldsymbol{I}_{12}^{T}= & {\left[\begin{array}{cccccc}
0 & r_{1}^{\prime} & s_{1}^{\prime} & t_{1}^{\prime} & u_{1}^{\prime} & v_{1}^{\prime} \\
-1 & r_{6}^{\prime} & s_{6}^{\prime} & t_{6}^{\prime} & u_{6}^{\prime} & v_{6}^{\prime} \\
0 & r_{5}^{\prime} & s_{5}^{\prime} & t_{5}^{\prime} & u_{5}^{\prime} & v_{5}^{\prime} \\
0 & r_{7}^{\prime} & s_{7}^{\prime} & t_{7}^{\prime} & u_{7}^{\prime} & v_{7}^{\prime} \\
0 & r_{15}^{\prime} & s_{15}^{\prime} & t_{15}^{\prime} & u_{15}^{\prime} & v_{15}^{\prime} \\
0 & r_{11}^{\prime} & s_{11}^{\prime} & t_{11}^{\prime} & u_{11}^{\prime} & v_{11}^{\prime}
\end{array}\right], } \\
\boldsymbol{N}_{2}^{(-1)}=-\boldsymbol{q}+\boldsymbol{r}_{1} \boldsymbol{t}_{1}^{-1} \boldsymbol{r}_{1}^{T}=- & {\left[\begin{array}{ccccccc}
\kappa_{11}^{\prime} & \kappa_{16}^{\prime} & \kappa_{15}^{\prime} & \kappa_{17}^{\prime} & \kappa_{115}^{\prime} & \kappa_{111}^{\prime} \\
\kappa_{16}^{\prime} & \kappa_{66}^{\prime} & \kappa_{56}^{\prime} & \kappa_{67}^{\prime} & \kappa_{615}^{\prime} & \kappa_{611}^{\prime} \\
\kappa_{15}^{\prime} & \kappa_{56}^{\prime} & \kappa_{55}^{\prime} & \kappa_{57}^{\prime} & \kappa_{515}^{\prime} & \kappa_{511}^{\prime} \\
\kappa_{17}^{\prime} & \kappa_{67}^{\prime} & \kappa_{57}^{\prime} & \kappa_{77}^{\prime} & \kappa_{715}^{\prime} & \kappa_{711}^{\prime} \\
\kappa_{115}^{\prime} & \kappa_{615}^{\prime} & \kappa_{515}^{\prime} & \kappa_{715}^{\prime} & \kappa_{1515}^{\prime} & \kappa_{115}^{\prime} \\
\kappa_{111}^{\prime} & \kappa_{611}^{\prime} & \kappa_{511}^{\prime} & \kappa_{711}^{\prime} & \kappa_{1115}^{\prime} & \kappa_{1111}^{\prime}
\end{array}\right], }
\end{aligned}
$$


where $\Delta^{\prime}$ is the determinant of

$$
\boldsymbol{W}^{\prime}=\left[\begin{array}{ccccc}
S_{22} & S_{24} & S_{212} & S_{28} & S_{213} \\
S_{24} & S_{44} & S_{412} & S_{48} & S_{413} \\
S_{212} & S_{412} & S_{1212} & S_{812} & S_{1213} \\
S_{28} & S_{48} & S_{812} & S_{88} & S_{813} \\
S_{213} & S_{413} & S_{1213} & S_{813} & S_{1313}
\end{array}\right],
$$

$\widehat{\boldsymbol{W}}^{\prime}=\left[\widehat{W}_{i j}^{\prime}\right]$ is the cofactor of $\boldsymbol{W}^{\prime}$, and

$$
\begin{aligned}
& r_{\alpha}^{\prime}=\frac{1}{\Delta^{\prime}}\left|\begin{array}{ccccc}
S_{2 \alpha} & S_{4 \alpha} & S_{12 \alpha} & S_{8 \alpha} & S_{13 \alpha} \\
S_{24} & S_{44} & S_{412} & S_{48} & S_{413} \\
S_{212} & S_{412} & S_{1212} & S_{812} & S_{1213} \\
S_{28} & S_{48} & S_{812} & S_{88} & S_{813} \\
S_{213} & S_{413} & S_{1213} & S_{813} & S_{1313}
\end{array}\right|, \quad s_{\alpha}^{\prime}=\frac{1}{\Delta^{\prime}}\left|\begin{array}{ccccc}
S_{22} & S_{24} & S_{212} & S_{28} & S_{213} \\
S_{2 \alpha} & S_{4 \alpha} & S_{12 \alpha} & S_{8 \alpha} & S_{13 \alpha} \\
S_{212} & S_{412} & S_{1212} & S_{812} & S_{1213} \\
S_{28} & S_{48} & S_{812} & S_{88} & S_{813} \\
S_{213} & S_{413} & S_{1213} & S_{813} & S_{1313}
\end{array}\right| \text {, } \\
& t_{\alpha}^{\prime}=\frac{1}{\Delta^{\prime}}\left|\begin{array}{lllll}
S_{22} & S_{24} & S_{212} & S_{28} & S_{213} \\
S_{24} & S_{44} & S_{412} & S_{48} & S_{413} \\
S_{2 \alpha} & S_{4 \alpha} & S_{12 \alpha} & S_{8 \alpha} & S_{13 \alpha} \\
S_{28} & S_{48} & S_{812} & S_{88} & S_{813} \\
S_{213} & S_{413} & S_{1213} & S_{813} & S_{1313}
\end{array}\right|, \quad u_{\alpha}^{\prime}=\frac{1}{\Delta^{\prime}}\left|\begin{array}{ccccc}
S_{22} & S_{24} & S_{212} & S_{28} & S_{213} \\
S_{24} & S_{44} & S_{412} & S_{48} & S_{413} \\
S_{212} & S_{412} & S_{1212} & S_{812} & S_{1213} \\
S_{2 \alpha} & S_{4 \alpha} & S_{12 \alpha} & S_{8 \alpha} & S_{13 \alpha} \\
S_{213} & S_{413} & S_{1213} & S_{813} & S_{1313}
\end{array}\right|, \\
& v_{\alpha}^{\prime}=\frac{1}{\Delta^{\prime}}\left|\begin{array}{ccccc}
S_{22} & S_{24} & S_{212} & S_{28} & S_{213} \\
S_{24} & S_{44} & S_{412} & S_{48} & S_{413} \\
S_{212} & S_{412} & S_{1212} & S_{812} & S_{1213} \\
S_{28} & S_{48} & S_{812} & S_{88} & S_{813}
\end{array}\right| \quad(\alpha=1,6,5,7,15,11), \\
& \begin{array}{lllll}
S_{28} & S_{48} & S_{812} & S_{88} & S_{813} \\
S_{2 \alpha} & S_{4 \alpha} & S_{12 \alpha} & S_{8 \alpha} & S_{13 \alpha}
\end{array} \mid \\
& \kappa_{\alpha \beta}^{\prime}=\frac{1}{\Delta^{\prime}}\left|\begin{array}{cccccc}
S_{\alpha \beta} & S_{2 \beta} & S_{4 \beta} & S_{12 \beta} & S_{8 \beta} & S_{13 \beta} \\
S_{2 \alpha} & S_{22} & S_{24} & S_{212} & S_{28} & S_{213} \\
S_{4 \alpha} & S_{24} & S_{44} & S_{412} & S_{48} & S_{413} \\
S_{12 \alpha} & S_{212} & S_{412} & S_{1212} & S_{812} & S_{1213} \\
S_{8 \alpha} & S_{28} & S_{48} & S_{812} & S_{88} & S_{813} \\
S_{13 \alpha} & S_{213} & S_{413} & S_{1213} & S_{813} & S_{1313}
\end{array}\right| \quad \quad(\alpha, \beta=1,6,5,7,15,11)
\end{aligned}
$$

In view of (12), the matrix $-N_{2}^{(-1)}$ is positive definite, while, by (24) and the fact that $\boldsymbol{W}^{\prime}$ defined by (27) is positive definite, we also have that $N_{3}^{(-1)}$ is positive semidefinite.

\section{Line force and line dislocation}

4.1. Line force and dislocation in a homogeneous quasicrystal. Let a line of uniformly distributed force $\boldsymbol{p}=\left[\begin{array}{llllll}p_{1} & p_{2} & p_{3} & q_{1} & q_{2} & q_{3}\end{array}\right]^{T}$ per unit length be applied on the $x_{3}$-axis which also includes a line dislocation with Burgers vector 
$\boldsymbol{b}=\left[\begin{array}{llllll}b_{1} & b_{2} & b_{3} & d_{1} & d_{2} & d_{3}\end{array}\right]^{T}$. The analytic vector function $\boldsymbol{f}(z)$ due to the line force and dislocation is given by

$$
\boldsymbol{f}(z)=\left\langle\ln z_{\alpha}\right\rangle \frac{\boldsymbol{A}^{T} \boldsymbol{p}+\boldsymbol{B}^{T} \boldsymbol{b}}{2 \pi \mathrm{i}},
$$

where $\langle *\rangle$ is a $6 \times 6$ diagonal matrix in which each component varies with the index $\alpha$ (from 1 to 6 ). The elastic energy for the annular region $0<r_{0} \leq r \leq R_{0}$ per unit length of the line force and dislocation is

$$
U=\frac{1}{4 \pi} \ln \left(\frac{R_{0}}{r_{0}}\right)\left(\boldsymbol{p}^{T} \boldsymbol{H} \boldsymbol{p}+\boldsymbol{b}^{T} \boldsymbol{L} \boldsymbol{b}\right)>0,
$$

which also provides an indirect proof that $\boldsymbol{H}$ and $\boldsymbol{L}$ must be positive definite if the elastic energy is to remain positive.

4.2. Interfacial Green's function. We consider a bimaterial made of two bonded dissimilar anisotropic quasicrystalline half-planes, denoted by \#1 $\left(x_{2}>0\right)$ and \#2 $\left(x_{2}<0\right)$. The bimaterial is subjected to a line force $\boldsymbol{p}$ and line dislocation with Burgers vector $\boldsymbol{b}$ at the origin. In fact the solution can be found from [Ting 1996]. The elastic energy for the annular region $0<r_{0} \leq r \leq R_{0}$ of the quasicrystalline bimaterial is

$$
U=\frac{1}{2 \pi} \ln \left(\frac{R_{0}}{r_{0}}\right)\left(\boldsymbol{p}^{T} \tilde{\boldsymbol{H}} \boldsymbol{p}+\boldsymbol{b}^{T} \tilde{\boldsymbol{L}} \boldsymbol{b}\right)>0,
$$

where $\tilde{\boldsymbol{H}}$ and $\tilde{\boldsymbol{L}}$ are two $6 \times 6$ positive definite real symmetric matrices given by

$$
\tilde{\boldsymbol{H}}=\operatorname{Re}\left\{\left(\boldsymbol{M}_{1}+\overline{\boldsymbol{M}}_{2}\right)^{-1}\right\}, \quad \tilde{\boldsymbol{L}}=\operatorname{Re}\left\{\left(\boldsymbol{M}_{1}^{-1}+\overline{\boldsymbol{M}}_{2}^{-1}\right)^{-1}\right\} .
$$

4.3. Green's function for quasicrystalline bimaterials. We consider a bimaterial made of two perfectly bonded dissimilar anisotropic quasicrystalline half-planes again denoted by \#1 $\left(x_{2}>0\right)$ and \#2 $\left(x_{2}<0\right)$. A line force $\boldsymbol{p}$ and line dislocation with Burgers vector $\boldsymbol{b}$ are applied at $\left(x_{1}, x_{2}\right)=(0, \delta)(\delta>0)$ in material \#1. The structure of the solution is similar to that in [Suo 1990] and [Ting 1996]. The image force tending to move a pure dislocation $(\boldsymbol{p}=\mathbf{0})$ away from the interface is described by

$$
F=\frac{1}{4 \pi \delta} \boldsymbol{b}^{T}\left(2 \tilde{\boldsymbol{L}}-\boldsymbol{L}_{1}\right) \boldsymbol{b}
$$

where $\tilde{\boldsymbol{L}}$ is given by (31). For a sliding interface on which $\sigma_{12}=\sigma_{32}=0$, the image force acting on the line dislocation is now characterized by

$$
F=\frac{1}{4 \pi \delta}\left(2 \boldsymbol{b}_{0}^{T} \hat{\boldsymbol{L}} \boldsymbol{b}_{0}-\boldsymbol{b}^{T} \boldsymbol{L}_{1} \boldsymbol{b}\right)
$$


where

$$
\boldsymbol{b}_{0}=\left[\begin{array}{llll}
b_{2} & d_{1} & d_{2} & d_{3}
\end{array}\right]^{T}, \quad \hat{\boldsymbol{L}}=\operatorname{Re}\left\{\hat{\boldsymbol{N}}^{-1}\right\}, \quad \hat{\boldsymbol{N}}=\left[\begin{array}{llll}
N_{22} & N_{24} & N_{25} & N_{26} \\
\bar{N}_{24} & N_{44} & N_{45} & N_{46} \\
\bar{N}_{25} & \bar{N}_{45} & N_{55} & N_{56} \\
\bar{N}_{26} & \bar{N}_{46} & \bar{N}_{56} & N_{66}
\end{array}\right],
$$

$N_{i j}$ being the components of the $6 \times 6$ Hermitian matrix $N$ defined by (14). Both $\hat{N}$ and $\hat{\boldsymbol{L}}$ are positive definite.

\section{Imperfect interface models}

In [Benveniste 2006], the author rigorously derives imperfect interface models for a three-dimensional curved interphase between two anisotropic crystalline solids by making use of the idea of Taylor expansion of the corresponding fields in thin regions. In this section we will derive compliant and stiff interface models in anisotropic quasicrystalline bimaterials using the Stroh formalism and the structures of $\boldsymbol{N}_{i}$ presented in Sections 2 and 3. To simplify the analysis, we consider here a straight imperfect interface. The stress-strain law for an interphase of constant thickness $h$ between the upper and lower anisotropic quasicrystalline materials 1 and 2 is described by

$$
\boldsymbol{\sigma}_{1}=\boldsymbol{Q}_{c} \boldsymbol{u}_{, 1}+\boldsymbol{R}_{c} \boldsymbol{u}_{, 2}, \boldsymbol{\sigma}_{2}=\boldsymbol{R}_{c}^{T} \boldsymbol{u}_{, 1}+\boldsymbol{T}_{c} \boldsymbol{u}_{, 2},
$$

where $\boldsymbol{Q}, \boldsymbol{R}, \boldsymbol{T}$ are defined in (7) with the subscript $c$ being used to identify the quantities associated with the intermediate interphase, and

$$
\sigma_{1}=\left[\begin{array}{llllll}
\sigma_{11} & \sigma_{21} & \sigma_{31} & H_{11} & H_{21} & H_{31}
\end{array}\right]^{T}, \sigma_{2}=\left[\begin{array}{llllll}
\sigma_{12} & \sigma_{22} & \sigma_{32} & H_{12} & H_{22} & H_{32}
\end{array}\right]^{T} .
$$

(1) If we assume that $C_{i j k l}^{(c)} \ll C_{i j k l}^{(1)}, C_{i j k l}^{(2)} ; R_{i j k l}^{(c)} \ll R_{i j k l}^{(1)}, R_{i j k l}^{(2)}$ and $K_{i j k l}^{(c)} \ll$ $K_{i j k l}^{(1)}, K_{i j k l}^{(2)}$ (the so-called compliant interphase) and that the interphase is also very thin, then it follows from $(35)_{2}$ that

$$
\boldsymbol{u}_{1}-\boldsymbol{u}_{2}=h \boldsymbol{N}_{2}^{(c)} \boldsymbol{\sigma}_{2}^{(1)}=h \boldsymbol{N}_{2}^{(c)} \boldsymbol{\sigma}_{2}^{(2)} \text { on the compliant interface. }
$$

This is, in fact, the anisotropic spring-type interface.

(2) If we assume that $C_{i j k l}^{(c)} \gg C_{i j k l}^{(1)}, C_{i j k l}^{(2)}$ and $K_{i j k l}^{(c)} \gg K_{i j k l}^{(1)}, K_{i j k l}^{(2)}$ (the so-called stiff interphase) and that the interphase is also very thin, then it follows from (35) and the equilibrium equation $\sigma_{1,1}+\sigma_{2,2}=\mathbf{0}$ that

$$
u_{1}=u_{2}, \quad \sigma_{2}^{(1)}-\sigma_{2}^{(2)}=h N_{3}^{(c)} u_{1,11}=h N_{3}^{(c)} u_{2,11} \text { on the stiff interface. }
$$

This is an extension of the Young-Laplace model to anisotropic quasicrystalline materials. 
In view of the structure of $N_{3}$ given by (18), the phonon normal traction component $\sigma_{22}$ is continuous across the stiff interface.

\section{Interface crack problems}

First we derive the asymptotic fields associated with a traction-free semi-infinite crack which lies along the interface between the upper and lower anisotropic quasicrystalline half-planes \#1 $\left(x_{2}>0\right)$ and \#2 $\left(x_{2}<0\right)$. The portion $x_{2}=0, x_{1}>0$ of the interface is perfectly bonded, whereas the remaining portion $x_{2}=0$ and $x_{1}<0$ of the interface is fully debonded. In the following analysis, in view of the fact that $z_{1}=z_{2}=z_{3}=z_{4}=z_{5}=z_{6}=z$ on the interface $x_{2}=0$, we will replace the complex variables $z_{k}(k=1, \ldots, 6)$ by the common complex variable $z=x_{1}+\mathrm{i} x_{2}$. After the analysis is completed, we can simply revert back to the corresponding complex variables.

We introduce an analytic vector function $\boldsymbol{h}(z)$ defined by

$$
\boldsymbol{h}(z)=\boldsymbol{B}_{1} \boldsymbol{f}_{1}^{\prime}(z)=\boldsymbol{N}^{-1} \overline{\boldsymbol{N}} \boldsymbol{B}_{2} \boldsymbol{f}_{2}^{\prime}(z) .
$$

Consequently the traction and displacement jumps can be expressed in terms of $\boldsymbol{h}(z)$ as

$$
\sigma_{2}\left(x_{1}\right)=\boldsymbol{h}^{+}\left(x_{1}\right)+\overline{\boldsymbol{N}}^{-1} \boldsymbol{N} \boldsymbol{h}^{-}\left(x_{1}\right), \quad \mathrm{i} \boldsymbol{d}^{\prime}\left(x_{1}\right)=\boldsymbol{N}\left[\boldsymbol{h}^{+}\left(x_{1}\right)-\boldsymbol{h}^{-}\left(x_{1}\right)\right],
$$

We then arrive at the following homogeneous vector Riemann-Hilbert problem:

$$
\begin{aligned}
\boldsymbol{h}^{+}(z)-\boldsymbol{h}^{-}(z) & =\mathbf{0}, \quad z \notin C, \\
\overline{\boldsymbol{N}} \boldsymbol{h}^{+}(z)+\boldsymbol{N} \boldsymbol{h}^{-}(z) & =\mathbf{0}, \quad z \in C .
\end{aligned}
$$

Consider the eigenvalue problem

$$
\bar{N} \boldsymbol{w}=\mathrm{e}^{2 \pi \epsilon} \boldsymbol{N} \boldsymbol{w} .
$$

It can be concluded that:

(1) As a result of the positive definiteness of $N$, the eigenvalue $e^{2 \pi \epsilon}$ is always positive; thus $\epsilon$ is real.

(2) If $(\epsilon, \boldsymbol{w})$ is an eigenpair, then $(-\epsilon, \overline{\boldsymbol{w}})$ is another eigenpair.

Three positive real numbers $\epsilon_{1}, \epsilon_{2}, \epsilon_{3}$ and three complex vectors $\boldsymbol{w}_{1}, \boldsymbol{w}_{2}, \boldsymbol{w}_{3}$ form six eigenpairs:

$$
\left(\epsilon_{1}, \boldsymbol{w}_{1}\right),\left(-\epsilon_{1}, \overline{\boldsymbol{w}}_{1}\right),\left(\epsilon_{2}, \boldsymbol{w}_{2}\right),\left(-\epsilon_{2}, \overline{\boldsymbol{w}}_{2}\right),\left(\epsilon_{3}, \boldsymbol{w}_{3}\right),\left(-\epsilon_{3}, \overline{\boldsymbol{w}}_{3}\right) .
$$

The following orthogonal relationships can also be established:

$$
\boldsymbol{w}_{i}^{T} \boldsymbol{N} \boldsymbol{w}_{j}=\overline{\boldsymbol{w}}_{k}^{T} \boldsymbol{N} \boldsymbol{w}_{l}=\boldsymbol{w}_{k}^{T} \boldsymbol{N} \overline{\boldsymbol{w}}_{l}=0 \quad(i, j, k, l=1,2,3 \text { and } k \neq l),
$$


The three positive real numbers (or oscillatory indices) $\epsilon_{1}, \epsilon_{2}, \epsilon_{3}$ are given by

$$
\epsilon_{j}=\frac{1}{2 \pi} \ln \frac{1+\beta_{j}}{1-\beta_{j}} \quad(j=1,2,3),
$$

where $\beta_{j}(j=1,2,3)$ are the three positive roots of the following cubic equation in $\beta^{2}$ :

$$
\beta^{6}+\frac{1}{2} \operatorname{tr}\left(\breve{\boldsymbol{S}}^{2}\right) \beta^{4}-\frac{1}{2}|\breve{\boldsymbol{S}}| \operatorname{tr}\left(\breve{\boldsymbol{S}}^{-2}\right) \beta^{2}-|\breve{\boldsymbol{S}}|=0
$$

Here

$$
\breve{S}=\left(\boldsymbol{L}_{1}^{-1}+\boldsymbol{L}_{2}^{-1}\right)^{-1}\left(\boldsymbol{S}_{1} \boldsymbol{L}_{1}^{-1}-\boldsymbol{S}_{2} \boldsymbol{L}_{2}^{-1}\right) .
$$

The oscillatory indexes $\epsilon_{1}, \epsilon_{2}, \epsilon_{3}$ are then explicitly determined.

Following Suo [1990], we can obtain for $\boldsymbol{h}(z)$ the expression

$$
\boldsymbol{h}(z)=\sum_{j=1}^{3} \frac{\mathrm{e}^{\pi \epsilon_{j}} K_{j} z^{\mathrm{i} \epsilon_{j}} \boldsymbol{w}_{j}+\mathrm{e}^{-\pi \epsilon_{j}} \bar{K}_{j} z^{-\mathrm{i} \epsilon_{j}} \overline{\boldsymbol{w}}_{j}}{2(2 \pi z)^{\frac{1}{2}} \cosh \pi \epsilon_{j}},
$$

where $K_{1}, K_{2}$ and $K_{3}$ are three complex stress intensity factors. $\boldsymbol{f}_{1}^{\prime}(z)$ and $\boldsymbol{f}_{2}^{\prime}(z)$ in the two half-planes are then given by

$$
\begin{aligned}
& \boldsymbol{B}_{1} \boldsymbol{f}_{1}^{\prime}(z)=\sum_{j=1}^{3} \frac{\mathrm{e}^{\pi \epsilon_{j}} K_{j} z^{\mathrm{i} \epsilon_{j}} \boldsymbol{w}_{j}+\mathrm{e}^{-\pi \epsilon_{j}} \bar{K}_{j} z^{-\mathrm{i} \epsilon_{j}} \overline{\boldsymbol{w}}_{j}}{2(2 \pi z)^{\frac{1}{2}} \cosh \pi \epsilon_{j}} \quad(\operatorname{Im}\{z\}>0), \\
& \boldsymbol{B}_{2} \boldsymbol{f}_{2}^{\prime}(z)=\sum_{j=1}^{3} \frac{\mathrm{e}^{-\pi \epsilon_{j}} K_{j} z^{\mathrm{i} \epsilon_{j}} \boldsymbol{w}_{j}+\mathrm{e}^{\pi \epsilon_{j}} \bar{K}_{j} z^{-\mathrm{i} \epsilon_{j}} \overline{\boldsymbol{w}}_{j}}{2(2 \pi z)^{\frac{1}{2}} \cosh \pi \epsilon_{j}} \quad(\operatorname{Im}\{z\}<0) .
\end{aligned}
$$

The traction at the bonded interface a distance $r$ ahead of the crack tip is

$$
\boldsymbol{\sigma}_{2}(r)=\sum_{j=1}^{3}\left[t_{j}(r) \boldsymbol{w}_{j}+\bar{t}_{j}(r) \overline{\boldsymbol{w}}_{j}\right]
$$

where

$$
t_{j}(r)=t_{2 j}+\mathrm{i} t_{1 j}=\frac{\overline{\boldsymbol{w}}_{j}^{T} \boldsymbol{N} \boldsymbol{\sigma}_{2}(r)}{\overline{\boldsymbol{w}}_{j}^{T} \boldsymbol{N} \boldsymbol{w}_{j}}=\frac{K_{j} r^{\mathrm{i} \epsilon_{j}}}{\sqrt{2 \pi r}} .
$$

Equation (50) states that the interface traction can be decomposed into three components, each in the plane spanned by $\operatorname{Re}\left\{\boldsymbol{w}_{j}\right\}$ and $\operatorname{Im}\left\{\boldsymbol{w}_{j}\right\}(j=1,2,3)$.

The displacement jump a distance $r$ behind the crack tip is

$$
\boldsymbol{d}(r)=(\boldsymbol{N}+\overline{\boldsymbol{N}})\left(\frac{r}{2 \pi}\right)^{\frac{1}{2}} \sum_{j=1}^{3}\left[\frac{K_{j} r^{\mathrm{i} \epsilon_{j}} \boldsymbol{w}_{j}}{\left(1+2 \mathrm{i} \epsilon_{j}\right) \cosh \pi \epsilon_{j}}+\frac{\bar{K}_{j} r^{-\mathrm{i} \epsilon_{j}} \overline{\boldsymbol{w}}_{j}}{\left(1-2 \mathrm{i} \epsilon_{j}\right) \cosh \pi \epsilon_{j}}\right]
$$


The energy release rate is therefore given by

$$
G=\lim _{\delta \rightarrow 0} \frac{1}{2 \delta} \int_{0}^{\delta} \boldsymbol{\sigma}_{2}^{T}(\delta-r) d(r) d r=\sum_{j=1}^{3} \frac{\overline{\boldsymbol{w}}_{j}^{T}(\boldsymbol{N}+\overline{\boldsymbol{N}}) \boldsymbol{w}_{j}}{4 \cosh ^{2} \pi \epsilon_{j}}\left|K_{j}\right|^{2}>0 .
$$

Next, we consider a set of collinear cracks between two dissimilar anisotropic quasicrystalline half-planes with prescribed traction $t_{0}\left(x_{1}\right)$ on the crack lines $C$. Suppose there are $n$ finite cracks in the intervals $\left(a_{j}, b_{j}\right)(j=1,2, \ldots, n)$ and two semi-infinite cracks $\left(-\infty, b_{0}\right)$ and $\left(a_{0},+\infty\right)$. The prescribed traction $\boldsymbol{t}_{0}\left(x_{1}\right)$ on the crack lines $C$ will result in the inhomogeneous Riemann-Hilbert vector problem:

$$
\boldsymbol{h}^{+}\left(x_{1}\right)+\overline{\boldsymbol{N}}^{-1} \boldsymbol{N} \boldsymbol{h}^{-}\left(x_{1}\right)=\boldsymbol{t}_{0}\left(x_{1}\right), \quad x_{1} \in C .
$$

In order to solve this, we follow the method in [Suo 1990] and [Suo et al. 1992] and write $\boldsymbol{h}(z)$ and $\boldsymbol{t}_{0}\left(x_{1}\right)$ in terms of their components using the eigenvector representation

$$
\begin{aligned}
\boldsymbol{h}(z) & =h_{1}(z) \boldsymbol{w}_{1}+h_{2}(z) \overline{\boldsymbol{w}}_{1}+h_{3}(z) \boldsymbol{w}_{2}+h_{4}(z) \overline{\boldsymbol{w}}_{2}+h_{5}(z) \boldsymbol{w}_{3}+h_{6}(z) \overline{\boldsymbol{w}}_{3}, \\
\boldsymbol{t}_{0}\left(x_{1}\right) & =t_{01}\left(x_{1}\right) \boldsymbol{w}_{1}+\bar{t}_{01}\left(x_{1}\right) \overline{\boldsymbol{w}}_{1}+t_{02}\left(x_{1}\right) \boldsymbol{w}_{2}+\bar{t}_{02}\left(x_{1}\right) \overline{\boldsymbol{w}}_{2}+t_{03}\left(x_{1}\right) \boldsymbol{w}_{3}+\bar{t}_{03}\left(x_{1}\right) \overline{\boldsymbol{w}}_{3} .
\end{aligned}
$$

As a result, (53) can be decoupled as follows:

$$
\left.\begin{array}{l}
h_{1}^{+}\left(x_{1}\right)+\mathrm{e}^{-2 \pi \epsilon_{1}} h_{1}^{-}\left(x_{1}\right)=t_{01}\left(x_{1}\right) \\
h_{2}^{+}\left(x_{1}\right)+\mathrm{e}^{+2 \pi \epsilon_{1}} h_{2}^{-}\left(x_{1}\right)=\bar{t}_{01}\left(x_{1}\right) \\
h_{3}^{+}\left(x_{1}\right)+\mathrm{e}^{-2 \pi \epsilon_{2}} h_{3}^{-}\left(x_{1}\right)=t_{02}\left(x_{1}\right) \\
h_{4}^{+}\left(x_{1}\right)+\mathrm{e}^{+2 \pi \epsilon_{2}} h_{4}^{-}\left(x_{1}\right)=\bar{t}_{02}\left(x_{1}\right) \\
h_{5}^{+}\left(x_{1}\right)+\mathrm{e}^{-2 \pi \epsilon_{3}} h_{5}^{-}\left(x_{1}\right)=t_{03}\left(x_{1}\right) \\
h_{6}^{+}\left(x_{1}\right)+\mathrm{e}^{+2 \pi \epsilon_{3}} h_{6}^{-}\left(x_{1}\right)=\bar{t}_{03}\left(x_{1}\right)
\end{array}\right\} \quad \text { for } x_{1} \in C,
$$

whose solution can be given simply by

$$
\begin{aligned}
& h_{1}(z)=\frac{\chi_{1}(z)}{2 \pi \mathrm{i}} \int_{C} \frac{t_{01}\left(x_{1}\right) d x_{1}}{\chi_{1}^{+}\left(x_{1}\right)\left(x_{1}-z\right)}+\chi_{1}(z) P_{1}(z), \\
& h_{2}(z)=\frac{\bar{\chi}_{1}(z)}{2 \pi \mathrm{i}} \int_{C} \frac{\bar{t}_{01}\left(x_{1}\right) d x_{1}}{\bar{\chi}_{1}^{+}\left(x_{1}\right)\left(x_{1}-z\right)}+\bar{\chi}_{1}(z) P_{2}(z), \\
& h_{3}(z)=\frac{\chi_{2}(z)}{2 \pi \mathrm{i}} \int_{C} \frac{t_{02}\left(x_{1}\right) d x_{1}}{\chi_{2}^{+}\left(x_{1}\right)\left(x_{1}-z\right)}+\chi_{2}(z) P_{3}(z), \\
& h_{4}(z)=\frac{\bar{\chi}_{2}(z)}{2 \pi \mathrm{i}} \int_{C} \frac{\bar{t}_{02}\left(x_{1}\right) d x_{1}}{\bar{\chi}_{2}^{+}\left(x_{1}\right)\left(x_{1}-z\right)}+\bar{\chi}_{2}(z) P_{4}(z), \\
& h_{5}(z)=\frac{\chi_{3}(z)}{2 \pi \mathrm{i}} \int_{C} \frac{t_{03}\left(x_{1}\right) d x_{1}}{\chi_{3}^{+}\left(x_{1}\right)\left(x_{1}-z\right)}+\chi_{3}(z) P_{5}(z),
\end{aligned}
$$




$$
h_{6}(z)=\frac{\bar{\chi}_{3}(z)}{2 \pi \mathrm{i}} \int_{C} \frac{\bar{t}_{03}\left(x_{1}\right) d x_{1}}{\bar{\chi}_{3}^{+}\left(x_{1}\right)\left(x_{1}-z\right)}+\bar{\chi}_{3}(z) P_{6}(z)
$$

where $\chi_{1}(z), \chi_{2}(z)$ and $\chi_{3}(z)$ are defined as

$$
\chi_{j}(z)=\prod_{k=0}^{n}\left(z-a_{k}\right)^{-\frac{1}{2}-\mathrm{i} \epsilon_{j}}\left(z-b_{k}\right)^{-\frac{1}{2}+\mathrm{i} \epsilon_{j}} \quad(j=1,2,3),
$$

and $P_{i}(z)(i=1, \ldots, 6)$ are polynomials in $z$ of order less than $n+1$ [Ting 1996].

\section{Conclusions}

In this paper all six $6 \times 6$ matrices $\boldsymbol{N}_{i}$ and $\boldsymbol{N}_{i}^{(-1)}(i=1,2,3)$ in the Stroh formalism of anisotropic elasticity for quasicrystals have been explicitly expressed in terms of the sixty-six reduced elastic compliances $S_{i j}=S_{j i}(i, j=1 \ldots 15$ and $i, j \neq$ $3,9,10,14)$. It is found that $N_{2}$ and $-N_{2}^{(-1)}$ are positive definite, whilst $-N_{3}$ and $N_{3}^{(-1)}$ are positive semidefinite.

In the study of Green's functions, we present the elastic energy expressions (29) for a line force and dislocation in a homogeneous quasicrystal and (30) for a line force and dislocation lying on a bi-quasicrystal interface, and obtain the image force on a dislocation near a perfect or sliding interface between two anisotropic quasicrystalline half-planes.

We also derive compliant and stiff interface models using the Stroh formalism. Green's function solutions for quasicrystalline bimaterials with imperfect interface can be further studied by using the method described in [Wang and Pan 2010].

Perhaps the most interesting conclusion from this research is that the interface crack-tip field consists of three two-dimensional oscillatory stress singularities $r^{-\frac{1}{2} \pm \mathrm{i} \epsilon_{j}}(j=1,2,3)$ characterized through the introduction of three complex stress intensity factors $K_{j}(j=1,2,3)$. We end by again noting the beauty and power of the Stroh formalism which is fully demonstrated here.

\section{Acknowledgements}

Wang W. was supported by Innovation Program of Shanghai Municipal Education Commission (No. 12ZZ058). Schiavone acknowledges the support of the Natural Sciences and Engineering Research Council of Canada.

\section{References}

[Barnett and Lothe 1973] D. M. Barnett and J. Lothe, "Synthesis of the sextic and the integral formalism for dislocations, Green's functions and surface waves in anisotropic elastic solids", Phys. Norv. 7 (1973), 13-19. 
[Benveniste 2006] Y. Benveniste, "A general interface model for a three-dimensional curved thin anisotropic interphase between two anisotropic media", J. Mech. Phys. Solids 54:4 (2006), 708734.

[Cheng and Reddy 2002] Z.-Q. Cheng and J. N. Reddy, "Octet formalism for Kirchhoff anisotropic plates”, R. Soc. Lond. Proc. Ser. A Math. Phys. Eng. Sci. 458:2022 (2002), 1499-1517.

[Clements 1971] D. L. Clements, "A crack between dissimilar anisotropic media", Int. J. Eng. Sci. 9:2 (1971), 257-265.

[Ding et al. 1995] D.-H. Ding, R. Wang, W. Yang, C. Hu, and Y. Qin, "Elasticity theory of straight dislocations in quasicrystals", Phil. Mag. Lett. 72:5 (1995), 353-359.

[Eshelby et al. 1953] J. D. Eshelby, W. T. Read, and W. Shockley, "Anisotropic elasticity with applications to dislocation theory", Acta Metall. 1:3 (1953), 251-259.

[Gao et al. 1992] H. J. Gao, M. Abbudi, and D. M. Barnett, "On interfacial crack-tip field in anisotropic elastic solids", J. Mech. Phys. Solids 40:2 (1992), 393-416.

[Hu et al. 2000] C. Hu, R. Wang, and D.-H. Ding, "Symmetry groups, physical property tensors, elasticity and dislocations in quasicrystals”, Rep. Progr. Phys. 63:1 (2000), 1-39.

[Lazar and Kirchner 2005] M. Lazar and H. O. K. Kirchner, "Cosserat (micropolar) elasticity in Stroh form", Int. J. Solids Struct. 42:20 (2005), 5377-5398.

[Lekhnitskii 1950] S. G. Lekhnitskii, Теория упругости анизотропоного тела, Moscow, Gosud. Izdat. Tekhn.-Teor. Lit., 1950. Translated as Theory of elasticity of an anisotropic body, Holden-Day, San Francisco, 1963.

[Ru 2001] C. Q. Ru, "A two-dimensional Eshelby problem for two bonded piezoelectric half-planes", R. Soc. Lond. Proc. Ser. A Math. Phys. Eng. Sci. 457:2008 (2001), 865-883.

[Shechtman et al. 1984] D. Shechtman, I. Blech, D. Gratias, and J. W. Cahn, "Metallic phase with long-range orientational order and no translational symmetry", Phys. Rev. Lett. 53 (1984), 19511953.

[Stroh 1958] A. N. Stroh, "Dislocations and cracks in anisotropic elasticity", Phil. Mag. (8) 3 (1958), 625-646.

[Suo 1990] Z. Suo, "Singularities, interfaces and cracks in dissimilar anisotropic media", Proc. Roy. Soc. London Ser. A 427:1873 (1990), 331-358.

[Suo et al. 1992] Z. Suo, C.-M. Kuo, D. M. Barnett, and J. R. Willis, "Fracture mechanics for piezoelectric ceramics", J. Mech. Phys. Solids 40:4 (1992), 739-765.

[Ting 1986] T. C. T. Ting, "Explicit solution and invariance of the singularities at an interface crack in anisotropic composites", Internat. J. Solids Structures 22:9 (1986), 965-983.

[Ting 1988] T. C. T. Ting, "Some identities and the structure of $\mathbf{N}_{i}$ in the Stroh formalism of anisotropic elasticity”, Quart. Appl. Math. 46:1 (1988), 109-120.

[Ting 1996] T. C. T. Ting, Anisotropic elasticity: Theory and applications, Oxford Engineering Science Series 45, Oxford University Press, New York, 1996.

[Ting and Schiavone 2010] T. C. T. Ting and P. Schiavone, "Uniform antiplane shear stress inside an anisotropic elastic inclusion of arbitrary shape with perfect or imperfect interface bonding", Internat. J. Engrg. Sci. 48:1 (2010), 67-77.

[Wang and Pan 2010] X. Wang and E. Pan, “Two-dimensional Eshelby's problem for two imperfectly bonded piezoelectric half-planes", Int. J. Solids Struct. 47:1 (2010), 148-160.

[Willis 1964] J. R. Willis, "Anisotropic elastic inclusion problems", Quart. J. Mech. Appl. Math. 17 (1964), 157-174. 
[Willis 1970] J. R. Willis, "Stress fields produced by dislocations in anisotropic media", Philo. Mag. 21:173 (1970), 931-949.

[Willis 1971] J. R. Willis, "Fracture mechanics of interfacial cracks", J. Mech. Phys. Solids 19:6 (1971), 353-368.

Received 23 Feb 2012. Accepted 5 Apr 2012.

XU WANG: xuwang_sun@hotmail.com

School of Mechanical and Power Engineering, East China University of Science and Technology, 130 Meilong Road, Shanghai 200237, China

Peter Schiavone: p.schiavone@ualberta.ca

Department of Mechanical Engineering, University of Alberta, 4-9 Mechanical Engineering Building, Edmonton, AB T6G 2G8, Canada

http://www.mece.ualberta.ca/ schiavone/schiavon.htm

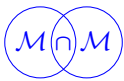


EDITORIAL BOARD

ANTONIO CARCATERRA ERIC A. CARLEN

FRANCESCO DELL' ISOLA

RAFFAELE ESPOSITO

ALBERT FANNJIANG

Gilles A. FrancForT

PiERANGelo MARCATI

JEAN-JACQUES MARIGo

PETER A. MARKOWICH

MARTIN OSTOJA-STARZEWSKI

PIERRE SEPPECHER

David J. SteigmanN

Paul STEINMANN

Pierre M. Suquet

\section{MANAGING EDITORS}

MICOL AMAR

CORRADO LATTANZIO

ANGELA MADEO

MARTIN OSTOJA-STARZEWSKI

\section{ADVISORY BOARD}

ADNAN AKAY

Holm AltenbaCH

MICOL AMAR

HARM ASKES

TEODOR ATANACKOVIĆ

VICTOR BERDICHEVSKY

GuY BouchitTÉ

ANDREA BRAIDES

ROBERTO CAMASSA

ERIC DARVE

Felix Darve

ANNA DE Masi

Gianpietro Del Piero

EMmanuele Di BenedetTo BERNOLD FIEDLER

IRENE M. GAMBA

SERGEy GavriLyUK

Timothy J. HEALEY

DOMINIQUE JEULIN ROGER E. KHAYAT

CorRado LATTANZIO ROBERT P. LIPTON

ANGELO LUONGO

ANGELA MADEO

JUAN J. MANFREDI

CARLO MARCHIORO

GÉrard A. Maugin

ROBERTO NATALINI

PATRIZIO NEFF

ANDREY PIATNITSKI

ERRICO PRESUTTI

MARIO PUlVIRENTI

LuCIO RusSO

Miguel A. F. SAnjuan

PATRICK SElVAdURA

ALEXANDER P. SEYRANIAN

MiroslaV ŠILHAVÝ

GUIDO SWEERS

ANTOINETTE TORDESILLAS

LEV TRUSKINOVSKY

JUAN J. L. VELÁZQUEZ

VINCENZO VESPRI

ANGELO VULPIANI msp.org/memocs

Università di Roma “La Sapienza”, Italia

Rutgers University, USA

(CO-CHAIR) Università di Roma "La Sapienza", Italia

(TREASURER) Università dell'Aquila, Italia

University of California at Davis, USA

(CO-CHAIR) Université Paris-Nord, France

Università dell'Aquila, Italy

École Polytechnique, France

DAMTP Cambridge, UK, and University of Vienna, Austria

(CHAIR MANAGING EDITOR) Univ. of Illinois at Urbana-Champaign, USA

Université du Sud Toulon-Var, France

University of California at Berkeley, USA

Universität Erlangen-Nürnberg, Germany

LMA CNRS Marseille, France

Università di Roma "La Sapienza”, Italia

Università dell' Aquila, Italy

Université de Lyon-INSA (Institut National des Sciences Appliquées), France (CHAIR MANAGING EDITOR) Univ. of Illinois at Urbana-Champaign, USA

Carnegie Mellon University, USA, and Bilkent University, Turkey

Otto-von-Guericke-Universität Magdeburg, Germany

Università di Roma "La Sapienza", Italia

University of Sheffield, UK

University of Novi Sad, Serbia

Wayne State University, USA

Université du Sud Toulon-Var, France

Università di Roma Tor Vergata, Italia

University of North Carolina at Chapel Hill, USA

Stanford University, USA

Institut Polytechnique de Grenoble, France

Università dell'Aquila, Italia

Università di Ferrara and International Research Center MEMOCS, Italia

Vanderbilt University, USA

Freie Universität Berlin, Germany

University of Texas at Austin, USA

Université Aix-Marseille, France

Cornell University, USA

École des Mines, France

University of Western Ontario, Canada

Università dell' Aquila, Italy

Louisiana State University, USA

Università dell' Aquila, Italia

Université de Lyon-INSA (Institut National des Sciences Appliquées), France

University of Pittsburgh, USA

Università di Roma "La Sapienza", Italia

Université Paris VI, France

Istituto per le Applicazioni del Calcolo "M. Picone", Italy

Universität Duisburg-Essen, Germany

Narvik University College, Norway, Russia

Università di Roma Tor Vergata, Italy

Università di Roma “La Sapienza”, Italia

Università di Roma "Tor Vergata", Italia

Universidad Rey Juan Carlos, Madrid, Spain

McGill University, Canada

Moscow State Lomonosov University, Russia

Academy of Sciences of the Czech Republic

Universität zu Köln, Germany

University of Melbourne, Australia

École Polytechnique, France

Bonn University, Germany

Università di Firenze, Italia

Università di Roma La Sapienza, Italia

MEMOCS (ISSN 2325-3444 electronic, 2326-7186 printed) is a journal of the International Research Center for the Mathematics and Mechanics of Complex Systems at the Università dell'Aquila, Italy.

Cover image: "Tangle” by @ John Horigan; produced using the Context Free program (contextfreeart.org).

\section{PUBLISHED BY}

\section{7 mathematical sciences publishers nonprofit scientific publishing}

http://msp.org/

(C) 2013 Mathematical Sciences Publishers 
Mathematics and Mechanics of Complex Systems vol. 1 no. 1

Dislocations, imperfect interfaces and interface cracks in anisotropic elasticity for quasicrystals

Xu Wang and Peter Schiavone

Localization of point vortices under curvature perturbations

Roberto Garra

Contraction of the proximal map and generalized convexity of the Moreau-Yosida regularization in the 2-Wasserstein metric

Eric A. Carlen and Katy Craig

Ptolemy's longitudes and Eratosthenes' measurement of the earth's circumference

\section{Lucio Russo}

TV-min and greedy pursuit for constrained joint sparsity and application to inverse scattering

Albert Fannjiang

On the theory of diffusion and swelling in finitely deforming elastomers

Gary J. Templet and David J. Steigmann

MEMOCS is a journal of the International Research Center for the Mathematics and Mechanics of Complex Systems at the Università dell' Aquila, Italy.

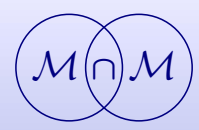

